\title{
Predicting ground state and metastable crystal structures using elemental and phonon mode descriptors
}

\author{
Aria Mansouri Tehrani, Bastien Grosso, Ramon Frey, Nicola A. Spaldin \\ Materials Theory, ETH Zurich, Wolfgang-Pauli-Strasse 27, 8093 Zürich, Switzerland \\ aria.mansouri.t@mat.ethz.ch
}

\begin{abstract}
We present a method to predict the crystal structure of any given composition using machine learning methods. Then, using the example of bismuth ferrite, we illustrate how crystal structure, decomposed into distortion modes, can be implemented as a feature to explore the energy surface leading to the identification of metastable polymorphs. Crystal structure plays a crucial role in determining the electronic structure and property of any composition. Therefore, it has always been of great interest to predict the crystal structure of any composition without requiring synthesis and characterization. To achieve this goal, we combine machine learning and density functional theory (DFT) calculations. Initially, a classification model predicts the point groups of the given stoichiometries. Based on the predicted point group, a series of high-throughput DFT calculations determine the ground state of non-centrosymmetric crystal structures. In addition to the ground state structure, identifying metastable polymorphs that might get stabilized by controlling the synthetic conditions is of great importance as they can exhibit different functionalities. Therefore, we studied $\mathrm{BiFeO}_{3}$ as a multifunctional compound with a rich low-energy phase space. A training set is constructed by mapping the phase space based on possible distortion modes starting from the cubic perovskite structure. A machine learning model is built using the generated training set predicting the energy surface of $\mathrm{BiFeO}_{3}$ to explore new metastable phases.
\end{abstract}

Keywords: Machine Learning; Bismuth Ferrite; Distortion Modes; DFT 